\title{
The association of sleep problems in children with autism and parental quality of life
}

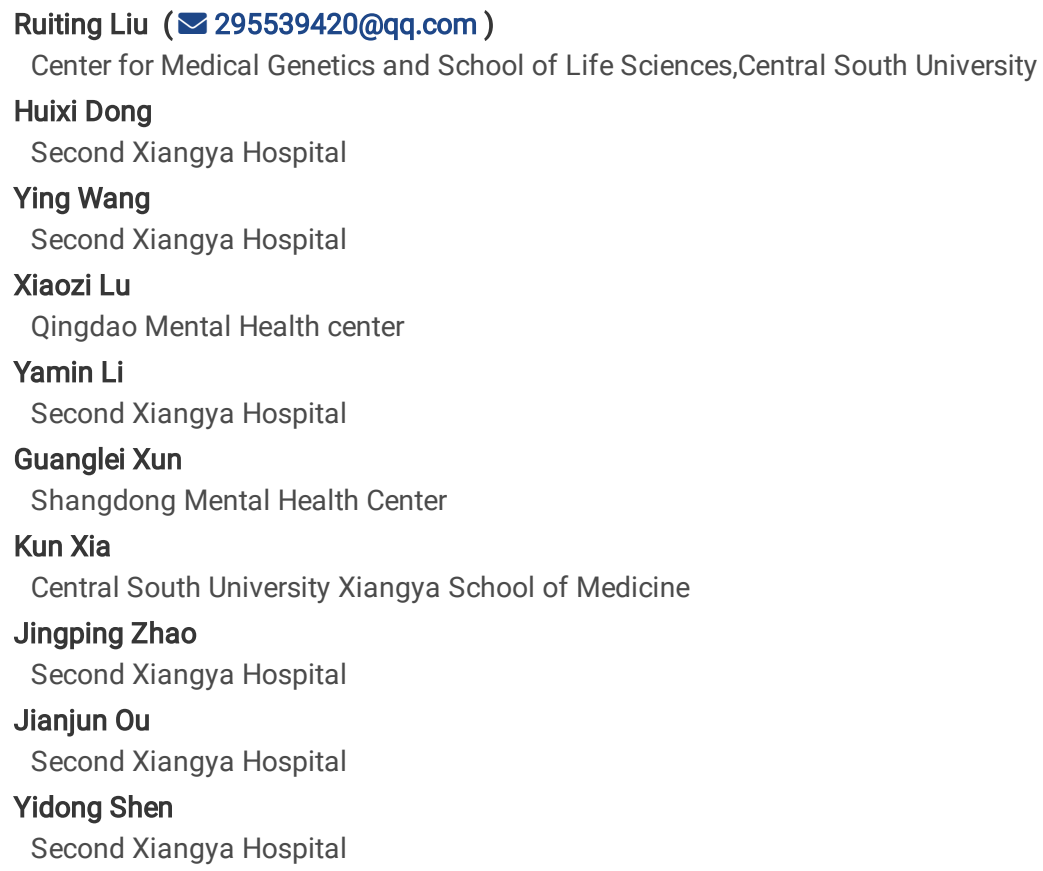




\section{Abstract}

Background Many children with autism suffer from sleep problems that impact not only children's daytime behavior and cognitive development but also their parents' life and health. The current study explored how and to what extent sleep problems in children with autism spectrum disorder (ASD) impacted their parents' quality of life (QOL). Methods This study adopted a case-control study design. A total of 440 ASD children aged 4 to 10 years and 344 agematched typically developing (TD) children were included in the study. The Children's Sleep Habits Questionnaire (CSHQ) was used to measure sleep problems in the enrolled children, and parental QOL was measured by the Short Form 36 Health Survey Questionnaire Version 2.0 (SF-36v2). Linear regression and pathway analysis were used to explore the impact of children's sleep problems on parental QOL. Results The linear regression model showed that the CSHQ total scores were negatively associated with maternal mental health summary (MCS) scores $(\beta=-2.831, p=0.005)$ in the TD group. In the ASD group, the CSHQ total scores were negatively associated with the parental physical health summary (PCS) scores ( $\beta=-3.030, p=0.003$ for mothers, $\beta=-3.651, p<0.001$ for fathers) after adjusting for the child's age, parental age, parental Zarit Caregiver Burden Interview (ZBI) score and Social Responsiveness Scale (SRS) score as covariates. The pathway analysis indicated that sleep problems in ASD children had both direct and indirect effects on maternal PCS. Conclusion The main results of the current study showed that sleep problems in children with or without ASD may affect parental QOL in different ways, and ASD children's sleep problems affect parental physical health as a risk factor independent from the social impairments associated with ASD and the childcare burden of the parents.

\section{Background}

Autism spectrum disorder (ASD) is a life-long neurodevelopmental disorder characterized by impairments in social interactions and stereotyped behaviors and interests with a childhood onset[1]. According to the most recent epidemiological survey conducted in the US, the prevalence of ASD has increased to $1.68 \%[2]$. Previous studies have indicated that some children with autism suffer from sleep problems[3], and these problems are more common in children with ASD than in typically developing (TD) children[4,5]. Parents have often reported that sleep-onset delay in children with ASD is one of the most concerning issues[6]. Other sleep problems, such as night waking and parasomnia, have also been frequently reported[7]. Sleep problems could be persistent in children with ASD and occur across different cognitive levels[6, 8]; numerous studies have found that sleep disturbances can affect daytime behaviors as well as core symptoms of ASD[9-11]. In addition to impacting children, sleep problems may also impact parents' health and well-being. Studies have reported that sleep disorders can affect the daily lives of older adults and lead to multiple comorbidities[12]. The effects of sleep disorders on quality of life (QOL) have been analyzed in patients with many diseases[13-15]. However, only a few studies have explored the effects of sleep problems in children with ASD on their parents. These limited studies indicated that sleep problems in children with ASD altered parental sleep quality[16] and caused increased stress in parents[17].

Previous studies have used the QOL concept to evaluate the impact of pervasive developmental disorders, including ASD, and other disorders or diseases on patients or their families. QOL is defined by the World Health Organization as the concept of an individual's perception of their own status in life in relation to the culture and the system of values in which they live and in relation to their goals, standards and needs[18]. It is a multidimensional concept involving an individual's physical and mental health, social relationships, personal beliefs and other aspects of life[19]. QOL of parents could affect their capacity to provide care and promote the health of their children and might also lead to better outcomes for the whole family. QOL may be affected by specific factors. For example, in one of our previous studies, we indicated that QOL in parents with children with ASD worsened with increasingly severe social impairments, and the impact of the social impairments on maternal QOL was mediated by childcare burden[20]. A study in South Africa also showed that parents of children with ASD had a significantly lower QOL than parents of TD children[21]. Thus, the QOL of parents of normal and autistic children is different, and sleep problems may be a factor.

We conducted the current study to explore how and to what extent the sleep problems in children with ASD impact their parents' QOL. We hypothesized that the impact of sleep problems in children with ASD on their parents' QOL may be different from that of TD children, and sleep problems are independent of the social impairments associated with ASD.

\section{Methods}

\section{Subjects}

This study adopted a case-control study design. Clinical data and information were extracted from a larger dataset developed in our previous research project that

was approved by the Human Ethics Committee of the Second Xiangya Hospital of Central South University. The children with ASD in the dataset were recruited from the Outpatient Department of the Mental Health Institute at the Second Xiangya Hospital of Central South University, Changsha, Hunan Province and four training schools for autistic children (the Elim Training Center for Children with Autism in Qingdao, Shandong Province, and the Xinyuan Training Center for Children with Autism, the Aimeng Training Center for Children with Autism, and the Qihang Training Center for Children with Autism in Changsha, Hunan province). The subjects in the control group were recruited from two kindergartens in Changsha. After obtaining written informed consent from the children's parents or their legal guardians, the children in the ASD group were independently diagnosed by two senior psychiatric doctors, according to DSM-IV-TR criteria for autistic disorder. The child's past illnesses were investigated, and children who had an organic disease of the nervous system or a psychiatric disorder, such as childhood schizophrenia or intellectual disability, were excluded from the study. Children with ASD with a chromosomal abnormality, such as Fragile X syndrome, and children with ASD and TD children whose parents had a disease were also excluded. 
Subjects aged 4 to 10 years in the dataset were selected. The Social Responsiveness Scale (SRS) was used as a complementary diagnostic test in the study, and a total raw score of 56.5 was set as the cutoff score[22]. Subjects who scored above 56.5 in the control group and scored below 56.5 in the case group were excluded. Ultimately, a total of 440 children with ASD and 344 age-matched TD children were included in the study. All of the included subjects were Han Chinese. In the case group, 383 subjects were male and 57 were female; their mean age was $5.13 \pm 1.12$ years. In the control group, 166 subjects were male and 178 were female; their mean age was $4.84 \pm 0.92$ years.

\section{Data collection and clinical assessment}

The parents of the children were asked to complete a self-administered structured questionnaire that included family demographic information. The questionnaire also included some widely used scales to measure some clinical features of the enrolled children and parents.

\subsection{Sleep problems}

The Children's Sleep Habits Questionnaire (CSHQ) was used to measure the sleep problems in the subjects in both groups. The CSHQ is a parent-reported questionnaire that uses a 3-point Likert scale and provides a total score and eight subdomain scores on bedtime resistance, sleep-onset delay, sleep duration, sleep anxiety, night waking, parasomnia, sleep-disordered breathing and daytime sleepiness. A higher score indicated more sleep problems. Based on the original report, the total score cutoff was set at 41[23]. However, some studies have indicated that a cutoff of 41 might overestimate sleep problems and suggested adopting the cutoff of 48 instead. Considering 48 as the cutoff value of the CSHQ can conservatively define sleep problems; thus, for the subsequent results, we considered a cutoff value of $48[5]$.

\subsection{Parental quality of life}

The Short Form 36 Health Survey Questionnaire Version 2.0 (SF-36v2) was adopted to measure parental QOL. The questionnaire categorizes QOL into 8 dimensions, including physical functioning, role-physical, pain, general health as energy/vitality, social functioning, role-emotional, and mental health. Two new comprehensive dimensions, the physical health summary (PCS) and mental health summary (MCS) scores, can be derived from the SF-36v2. A higher score on the SF-36v2 indicated better QOL. In the current study, we used a Chinese norm (Hong Kong) to provide the T scores for the SF-36v2[24], and the internal consistency and test-retest reliability of the Chinese form of the SF-36v2 is acceptable (coefficients 0.66 to 0.89 ).

\subsection{Childcare burden}

The Zarit Caregiver Burden Interview (ZBI) was used to measure childcare burden in the parents. Lu L et al.[25] translated the ZBI into Chinese from the English and Japanese versions, and according to their report, the internal consistency of the Chinese version of the ZBI was 0.875 . The ZBI contains 22 items scored on a 5-point Likert scale to measure the health, psychological well-being, social life, finances of the caregivers, and the relationship between caregivers and patients. Higher ZBI scores represented heavier childcare burden.

\subsection{Social impairments in ASD}

In addition to being used as a complementary diagnostic test, the SRS was also adopted to measure social impairments in the children with ASD. The SRS was developed to assess social behaviors in children[26]. The SRS is a parent-reported 65-item questionnaire that uses a 4-point Likert scale and includes five subdomains: awareness, cognition, communication, motivation, and autistic mannerisms. It is a widely used scale in autism research. It has convergent validity with the Autism Diagnostic Observation Schedule and the Autism Diagnostic Interview-Revised, ranging from 0.35 to 0.58 , and external validity with the Vinland Adaptive Behavior Scale and the Child Behavior Checklist, ranging from 0.35 to 0.64 [27]. A higher score on the SRS indicates greater impairments in social behavior.

\section{Statistical analysis}

We used Student's t-test to compare the demographic characteristics, children's CSHQ scores, and parental SF-36v2 scores between groups. A chi-square test was used to compare the incidence of sleep problems between the ASD and TD children. To test the impact of children's sleep problems on their parents' QOL, two linear regression models were used. The first linear regression model adjusted for the child's age, parental age and childcare burdens as covariates and was separately conducted in the ASD and TD groups. The second model adjusted SRS scores in the ASD group based on the first model to test whether sleep problems in children with ASD impacted parental QOL independent of social impairments. Additionally, if the second linear regression model found that the CSHQ scores had a significant effect on parental QOL, a pathway analysis would be conducted to explore the relationships among sleep problems, social impairments, childcare burden and parental QOL. As mentioned above, our previous study indicated that the social impairment associated with ASD affected parents' QOL due to the parental childcare burden. Considering that sleep problems in children can affect their social functioning [10] and increase the childcare burden on their parents [28], we hypothesized that sleep problems (CSHQ score) will exert direct impacts on parental QOL (SF-36v2 score), while social impairment (SRS score) and childcare burden (ZBI score) may act as mediators between parental QOL and sleep problems. An initial pathway recursive model was constructed based on our hypothesis and the findings of a previous study. The CSHQ score was characterized as an exogenous variable, and the SRS score, ZBI score and SF-36v2 score were characterized as endogenous variables.

All statistical analyses were conducted in IBM SPSS Statistics (Version 22.0) and IBM SPSS AMOS (Version 24.0). The significance level of all tests in the study was set at $p<0.05$ (two-tailed).

\section{Results}




\section{Comparisons of demographic information}

As shown in Table 1, the comparisons of the demographic information of the enrolled trios showed that there was a statistically significant but minor difference in the child's mean age between the case and control groups $(5.13 \pm 1.12$ vs. $4.84 \pm 0.92, t=4.05, p<0.001)$. The sex ratio of children in the ASD group was significantly different from that in the TD group $(\chi 2=138.39, p<0.001)$. There was no difference in the mean age of the parents $(p>0.05)$.

\section{Comparisons of the SRS, ZBI and SF-36v2}

Overall, the total raw SRS scores of the children with ASD were significantly higher than those of the TD children. The comparison of the ZBI showed that the fathers and mothers of children with ASD had a significantly higher ZBI score than the parents with TD children. The comparison of the SF-36v2 showed that both parents of children with ASD had a significantly lower SF-36v2 score than the parents of TD children in the PCS score and the MCS score. The details of comparisons of the SRS, ZBI and SF-36v2 scores are shown in Table 2.

\section{Comparisons of CSHQ}

The mean CSHQ score of the children with ASD was $48.26 \pm 6.99$ and that of the TD children was $46.99 \pm 6.71$. The difference in the mean score between the two groups was significant $(p=0.009)$. Details for each subdomain of the CSHQ are presented in Table 3 . When the cutoff score of 48 was adopted, the incidence of sleep problems in the children with ASD was $44.5 \%$, while the incidence was $34.0 \%$ in the TD children, and this difference reached a significant level $(\chi 2=8.932, p=0.003)$

\section{Impact of children's sleep problems on parental QOL}

The subjects were classified as good sleepers and poor sleepers based on the cutoff of 48[5]. The parental SF-36v2 scores were compared between the good sleepers and poor sleepers (Table 4). In the ASD group, the parents of the good sleepers had a significantly higher PCS score than the parents of the poor sleepers, but there was no significant difference in the MCS scores. In the TD group, the MCS scores of the parents of the good sleepers were significantly higher than those of the parents of the poor sleepers, while there was no significant difference in the PCS scores between the good and poor sleepers.

As shown in Table 5, the first linear regression model that adjusted for the child's age, parental age and childcare burden as covariates showed that the CSHQ total scores were negatively associated with the parental PCS scores $(\beta=-3.029, p=0.003$ for mothers; $\beta=-3.889, p<0.001$ for fathers) in the ASD group and negatively associated with maternal MCS scores $(\beta=-2.831, p=0.005)$ in the TD group. The second linear regression model conducted in the ASD group showed that the CSHQ scores were still negatively associated with the parental PCS scores $(\beta=-3.030, p=0.003$ for mothers; $\beta=-3.651, p<0.001$ for fathers) after adjusting for the child's age, parental age, parental ZBI scores and SRS scores as covariates.

\section{Pathway analysis}

The initial models and diagrams of the pathway analyses are shown in Figure 1 and Figure 2. Associations among the sleep CSHQ score, SRS score, ZBI score and parental PCS score were explored (father and mother).

For the mothers of the children with ASD, the initial model showed that sleep problems directly affected maternal PCS (pathway coefficient=-0.141, $p=0.003$, after adjustment in the multivariate statistical analysis, $p=0.018$ ), and that sleep problems also had an indirect effect on maternal PCS with mediation by social impairment and childcare burden (bold line in figure 1). In other words, more sleep problems were correlated with more serious social impairments (pathway coefficient $=0.161, p<0.001$ ), which may have caused a heavier childcare burden ( $p$ athway coefficient $=0.254, p<0.001$ ), and the childcare burden would finally affect the maternal PCS (pathway coefficient $=-0.163, p<0.001$ ). The initial model was a just-identified model that presented an acceptable fit to the data $(\mathrm{CFI}=1.000, \mathrm{NFI}=1.000$, RMSEA $=0.114)$. After adjusting the pathway model by deleting the nonsignificant pathway, the model was overidentified, the remaining pathway remained significant, and the fit indices improved $(X 2 / \mathrm{df}=0.917, \mathrm{CFI}=1.000, \mathrm{NFI}=0.973, \mathrm{TLI}=1.015$, RMSEA $=0.000)$.

For the fathers of the children with ASD, sleep problems of the children also had a direct effect on their PCS (pathway coefficient $=-0.183$, $p<0.001$ ). Unlike the mothers, the indirect effect pathway from child's sleep problem to paternal PCS was not found in the model (all ps>0.05).

\section{Discussion}

This case-control study explored the impact of sleep problems on children's parents. The main results of this study supported our hypothesis that sleep problems in children with ASD would affect parental physical health as an independent risk factor, and sleep problems of children in the ASD group may affect different aspects of parental QOL relative to those of TD children.

Here, we found that the incidence of sleep problems and the mean CSHQ scores were higher in the children with ASD than in the TD children, and these results were consistent with most previous studies[4, 5]. The reported incidence of sleep problems in children with ASD ranged from 40\% to $80 \%[29]$, while it was approximately $25 \%$ in TD children[5,23]. In our study, we used the CSHQ to examine the sleep quality of the enrolled subjects and adopted a cutoff score of 48 to define sleep problems. Reynolds et al[5] suggested that the original cutoff score of 41 might overidentify sleep problems in young children with ASD, so they used the cutoff score of 48 , which was based on the score defining the top 25th percentile in the general population. In their study, the reported incidence of sleep problems was $46.9 \%$ in children with ASD and that result was very similar to ours. The cause of the high incidence of sleep 
problems in children with ASD is not very clear. Several studies have indicated that many neurobiological causes, such as brain wave organization and maturation differences, abnormal melatonin production, and dysregulation of arousal and sensory systems, could make children with ASD vulnerable to sleep problems[6], which suggests that sleep problems may be an intrinsic feature of ASD.

Sleep problems will not only impact children's daytime behavior and cognitive development but also their parents' life and health. Studies examining the impact of sleep problems associated with ASD (or other neurodevelopmental disorders) on their parents are limited. In our study, we explored how and to what extent sleep problems in children with ASD impacted parental QOL. We found that sleep problems in children with ASD affected the physical health of the parents as a risk factor independent from the social impairments associated with ASD and the childcare burden of the parents. We assume that the relationship between children's sleep problems and parental physical health was accounted for by the long-term low quality of parental sleep. This is only a hypothesis, although some previous studies have made similar assumptions. Although we have not yet confirmed this hypothesis, future work will evaluate it in greater depth. Although our study did not examine the impact of children's sleep problems on parental sleep quality, previous studies have reported that sleep problems in children may cause more sleep difficulties in parents[20]. Our study also suggested that improving children's sleep quality may also improve parental QOL. Compared to social impairments associated with ASD, sleep problems are a pharmacologically treatable target symptom, and treatment or intervention goals for sleep may be easier to achieve than those for social impairments[30]. Therefore, additional studies on the effects of intervention or therapy for sleep problems in children with ASD are needed in the future.

On the other hand, our study found that sleep problems in children without ASD affected maternal mental health, which was different from the results in the parents of children with ASD, and this finding supported our hypothesis that the sleep problems of children with or without ASD may affect parental QOL in different ways. We assumed this difference may be because the parents of children with ASD paid more attention and spent more time coping with the children's social function deficits and daytime challenging behaviors, and the sleep problems may be relatively less prominent for them, while the parents of TD children may experience more stress from the sleep problems of their children.

Interestingly, our results indicated that sleep problems in TD children did not affect their father's PCS or MCS scores, and these results suggested a different child care pattern between mothers and fathers; perhaps, just as most mothers in China have often complained, fathers may be less involved in taking care of their children at night. This situation may be partly influenced by traditional Chinese culture, which has encouraged mothers to spend more time taking care of children in the family.

There are some limitations of our study. First, the sample size of this study is relatively small, which makes the statistical power not sufficient for testing the association between each subdomain score of the CSHQ and the parental QOL score. Second, in our linear regression model and pathway analysis, we did not consider whether the children with ASD were comorbid with attention-deficit/hyperactivity disorder (ADHD) or intellectual disability (ID), which may also be related to sleep problems in children. Finally, the SRS has a tendency to report anxiety; therefore, excluding TD individuals who report anxiety and ASD patients who report low anxiety risks results in losing valuable data and straying from research goals.

\section{Conclusions}

Our study explored the impact of sleep problems in children with or without ASD on parental QOL. The main findings of our study showed that sleep problems in children with or without ASD can affect parental QOL in different ways, and sleep problems in children with ASD could affect parental physical health as a risk factor independent from the social impairments associated with ASD and the childcare burden of the parents. The current study extends previous findings and suggests that improving children's sleep quality may also improve parental QOL. However, given some limitations of the study, more precise studies are still needed.

\section{Declarations}

\section{Ethics approval and consent to participate}

This study was a part of a large research project that was approved by the Human Ethics Committee of the Second Xiangya Hospital of Central South University. Written informed consent was obtained from all enrolled families.

\section{Consent for publication}

Not applicable. No individual-level data are presented in this publication.

\section{Availability of data and materials}

The datasets analyzed during the current study are available from the corresponding author upon reasonable request.

\section{Competing interests}

All authors declare that they have no competing interests.

\section{Funding}

This study was supported by the National Basic Research Program of China (Grant No. 2012CB517901) and the National Natural Science Foundation of China (Grant No. 81974217هNo.81601197). 


\section{Authors' contributions}

LR, DH, OJ, SY, and ZJ designed the study. LR performed the data analyses and wrote the draft of this manuscript. LR, DH, WY, LX, LY, XG, OJ, SY, XK, ZF, and ZJ managed data collection and contributed to data input. All authors contributed to the editing of the manuscript and approved the final text.

\section{Acknowledgments}

We thank all the subjects who participated in this study.

\section{References}

1. Lord, C., et al., Autism spectrum disorder. Lancet, 2018. 392(10146): p. 508-520.

2. Baio, J., et al., Prevalence of Autism Spectrum Disorder Among Children Aged 8 Years - Autism and Developmental Disabilities Monitoring Network, 11 Sites, United States, 2014. MMWR Surveill Summ, 2018. 67(6): p. 1-23.

3. Wang, G., et al., Sleep Disturbances and Associated Factors in Chinese Children with Autism Spectrum Disorder: A Retrospective and Cross-Sectional Study. Child Psychiatry Hum Dev, 2016. 47(2): p. 248-58.

4. Souders, M.C., et al., Sleep behaviors and sleep quality in children with autism spectrum disorders. Sleep, 2009. 32(12): p. 1566-78.

5. Reynolds, A.M., et al., Sleep Problems in 2- to 5-Year-Olds With Autism Spectrum Disorder and Other Developmental Delays. Pediatrics, 2019. 143(3).

6. Souders, M.C., et al., Sleep in Children with Autism Spectrum Disorder. Curr Psychiatry Rep, 2017. 19(6): p. 34.

7. Veatch, O.J., et al., Sleep in Children With Autism Spectrum Disorders: How Are Measures of Parent Report and Actigraphy Related and Affected by Sleep Education? Behav Sleep Med, 2016. 14(6): p. 665-76.

8. Goldman, S.E., et al., Parental sleep concerns in autism spectrum disorders: variations from childhood to adolescence. J Autism Dev Disord, 2012. 42(4): p. 531-8.

9. Mazzone, L., et al., The Relationship between Sleep Problems, Neurobiological Alterations, Core Symptoms of Autism Spectrum Disorder, and Psychiatric Comorbidities. J Clin Med, 2018. 7(5).

10. Veatch, O.J., et al., Shorter sleep duration is associated with social impairment and comorbidities in ASD. Autism Res, 2017. 10(7): p. 1221-1238.

11. Johnson, C.R., et al., Exploring sleep quality of young children with autism spectrum disorder and disruptive behaviors. Sleep Med, 2018. 44: p. 61-66.

12. Lin, J. and M. Suurna, Sleep Apnea and Sleep-Disordered Breathing. Otolaryngol Clin North Am, 2018. 51(4): p. $827-833$.

13. He, S., et al., Sleep disturbance, negative affect and health-related quality of life in patients with maintenance hemodialysis. Psychol Health Med, 2019. 24(3): p. 294-304

14. Iscar-Urrutia, M., et al., Objective and Subjective Sleep Efficiency in Adult Patients with Cystic Fibrosis and Impact on Quality of Life. Lung, 2018. 196(6): p. 761-767.

15. Pandey, S., et al., Impact of sleep quality on the quality of life of patients with Parkinson's disease: a questionnaire based study. Clin Neurol Neurosurg, 2016. 148: p. 29-34.

16. Lopez-Wagner, M.C., et al., Sleep problems of parents of typically developing children and parents of children with autism. J Genet Psychol, 2008. 169(3): p. 245-59.

17. Hodge, D., et al., Relationship between children's sleep and mental health in mothers of children with and without autism. J Autism Dev Disord, 2013. 43(4): p. 956-63.

18. The World Health Organization Quality of Life assessment (WHOQOL): position paper from the World Health Organization. Soc Sci Med, 1995. 41(10) p. 1403-9.

19. Mugno, D., et al., Impairment of quality of life in parents of children and adolescents with pervasive developmental disorder. Health Qual Life Outcomes, 2007. 5: p. 22.

20. Wang, Y., et al., Social impairment of children with autism spectrum disorder affects parental quality of life in different ways. Psychiatry Res, 2018. 266: p. 168-174.

21. Alhazmi, A., R. Petersen, and K.A. Donald, Quality of life among parents of South African children with autism spectrum disorder. Acta Neuropsychiatr, 2018. 30(4): p. 226-231

22. Cen, C.Q., et al., Investigating the validation of the Chinese Mandarin version of the Social Responsiveness Scale in a Mainland China child population BMC Psychiatry, 2017. 17(1): p. 51

23. Owens, J.A., A. Spirito, and M. McGuinn, The Children's Sleep Habits Questionnaire (CSHQ): psychometric properties of a survey instrument for schoolaged children. Sleep, 2000. 23(8): p. 1043-51.

24. Lam ETP, Lam CLK, Lo YYC, Gandek B. Psychometrics and population norm of the Chinese. (HK) SF-36 Health Survey:rsion 2. Hong Kong Practitioner. 2008;30П.

25. Lu, L., et al., Zarit Caregiver Burden Interview: development, reliability and validity of the Chinese version. Psychiatry Clin Neurosci, 2009. 63(6): p. 7304.

26. Constantino, J.N., et al., Reciprocal social behavior in children with and without pervasive developmental disorders. J Dev Behav Pediatr, 2000. 21(1): p. 2-11.

Page 6/11 
27. Bolte, S., F. Poustka, and J.N. Constantino, Assessing autistic traits: cross-cultural validation of the social responsiveness scale (SRS). Autism Res, 2008. 1(6): p. 354-63.

28. Tietze, A.L., et al., Sleep disturbances in children, adolescents, and young adults with severe psychomotor impairment: impact on parental quality of life and sleep. Dev Med Child Neurol, 2014. 56(12): p. 1187-1193.

29. Cohen, S., et al., The relationship between sleep and behavior in autism spectrum disorder (ASD): a review. J Neurodev Disord, 2014. 6(1): p. 44.

30. Cuomo, B.M., et al., Effectiveness of Sleep-Based Interventions for Children with Autism Spectrum Disorder: A Meta-Synthesis. Pharmacotherapy, 2017. 37(5): p. 555-578.

\section{Tables}

Table 1 Comparisons of demographic information of enrolled trios.

\begin{tabular}{|c|c|c|c|c|c|c|}
\hline & \multicolumn{2}{|c|}{$\begin{array}{l}\text { ASD group } \\
\text { Total } \mathrm{N}=440\end{array}$} & \multicolumn{2}{|c|}{$\begin{array}{c}\text { TD group } \\
\text { Total N=344 }\end{array}$} & \multirow[t]{2}{*}{$t / \chi^{2}$} & \multirow[t]{2}{*}{ Pvalue } \\
\hline & Mean or N & SD or \% & Mean or N & SD or $\%$ & & \\
\hline Child's age(years) & 5.13 & 1.12 & 4.84 & 0.92 & 4.05 & $<0.001$ \\
\hline Paternal age(years) & 35.10 & 4.43 & 35.13 & 5.00 & 0.10 & 0.920 \\
\hline Maternal age(years) & 32.89 & 3.63 & 32.35 & 4.12 & -1.91 & 0.057 \\
\hline \multicolumn{7}{|l|}{ Child's gender } \\
\hline Male(N) & 383 & 87.0 & 166 & 48.3 & 138.39 & $<0.001$ \\
\hline Female(N) & 57 & 13.0 & 178 & 51.7 & & \\
\hline
\end{tabular}

Table 2 Comparisons of SRS, ZBI and SF-36v2 scores between case and control group.

\begin{tabular}{lcccccccccc}
\hline & \multicolumn{3}{c}{ ASD group } & & \multicolumn{3}{c}{ TD group } & t & P value \\
\cline { 2 - 3 } \cline { 7 - 8 } & $\mathrm{n}$ & Mean & SD & & $\mathrm{n}$ & Mean & SD & & \\
\hline SRS score & 440 & 98.89 & 21.66 & & 344 & 41.50 & 9.91 & 49.36 & $<0.001$ \\
Paternal ZBI score & 440 & 31.20 & 15.49 & & 344 & 17.40 & 9.15 & 15.54 & $<0.001$ \\
Maternal ZBI score & 440 & 43.35 & 15.63 & & 344 & 17.77 & 10.08 & 27.72 & $<0.001$ \\
Paternal PCS score & 354 & 49.54 & 11.50 & & 327 & 55.29 & 7.13 & -7.908 & $<0.001$ \\
Paternal MCS score & 354 & 30.31 & 10.29 & & 327 & 48.12 & 10.29 & -13.31 & $<0.001$ \\
Maternal PCS score & 437 & 46.89 & 11.43 & & 343 & 53.13 & 8.36 & -9.362 & $<0.001$ \\
Maternal MCS score & 437 & 30.15 & 16.37 & & 343 & 47.21 & 12.27 & -16.64 & $<0.001$ \\
\hline
\end{tabular}

SRS Social Responsiveness Scales, ZBI The Zarit Caregiver Burden Interview, SF-36v2 The Short Form 36 Health Survey Questionnaire Version 2.0, PCS physical health summary, MCS mental health summary

Table 3 Comparisons of total score and each subdomain score of CSHQ between case and control group.

\begin{tabular}{lccccccc}
\hline & \multicolumn{2}{c}{ ASD group } & & \multicolumn{2}{c}{ TD group } & $\mathrm{t}$ & P value \\
& \multicolumn{2}{c}{$\mathrm{N}=440$} & & \multicolumn{2}{c}{$\mathrm{N}=344$} & & \\
\cline { 2 - 3 } & Mean & SD & & Mean & SD & & \\
\hline CSHQ total score & 48.27 & 6.99 & & 46.99 & 6.71 & 2.61 & 0.009 \\
Bed time resistance & 11.22 & 2.28 & & 10.51 & 2.13 & 4.43 & $<0.001$ \\
Sleep onset delay & 1.74 & 0.76 & & 1.62 & 0.70 & 2.36 & 0.018 \\
Sleep duration & 5.04 & 1.78 & & 4.94 & 1.63 & 0.82 & 0.412 \\
Sleep anxiety & 7.04 & 1.92 & & 6.70 & 1.98 & 2.41 & 0.016 \\
Night wakings & 3.68 & 1.10 & & 3.60 & 1.07 & 1.12 & 0.260 \\
Parasomnias & 8.78 & 1.94 & & 8.56 & 2.03 & 1.57 & 0.116 \\
Sleep disordered breathing & 3.45 & 0.92 & & 3.34 & 0.89 & 1.73 & 0.084 \\
Daytime Sleepiness & 11.57 & 2.59 & & 11.66 & 2.57 & -0.49 & 0.624 \\
\hline
\end{tabular}

CSHQ, Children's Sleep Habits Questionnaire

Table 4 Comparison of parental SF-36v2 score between good sleepers and bad sleepers ${ }^{\mathrm{a}}$ 


\begin{tabular}{|c|c|c|c|c|c|c|c|c|c|c|c|c|c|c|c|}
\hline & & \multirow[t]{3}{*}{$\mathrm{n}$} & \multicolumn{6}{|c|}{ Paternal SF-36 } & \multirow[t]{3}{*}{$\mathrm{n}$} & \multicolumn{6}{|c|}{ Maternal SF-36 } \\
\hline & & & PCS score & $\mathrm{t}$ & $\mathrm{P}$ & MCS score & $\mathrm{t}$ & $\mathrm{P}$ & & PCS score & $\mathrm{t}$ & $\mathrm{P}$ & MCS score & $\mathrm{t}$ & P value \\
\hline & & & Mean $\pm S D$ & & & Mean $\pm S D$ & & & & Mean $\pm S D$ & & & Mean \pm SD & & \\
\hline \multirow[t]{2}{*}{ ASD } & $\mathrm{TS}>48$ & 159 & $48.16 \pm 11.72$ & 2.05 & 0.041 & $29.09 \pm 23.33$ & 0.91 & 0.364 & 195 & $45.16 \pm 11.37$ & 2.20 & 0.029 & $29.09 \pm 16.34$ & 1.22 & 0.225 \\
\hline & $\mathrm{TS} \leq 48$ & 195 & $50.67 \pm 11.21$ & & & $31.31 \pm 22.36$ & & & 242 & $47.56 \pm 11.39$ & & & $31.00 \pm 16.38$ & & \\
\hline $\mathrm{TD}$ & $\mathrm{TS}>48$ & 108 & $54.14 \pm 6.83$ & 2.06 & 0.400 & $46.52 \pm 9.97$ & 1.99 & 0.047 & 117 & $52.00 \pm 9.28$ & 1.79 & 0.075 & $43.76 \pm 14.82$ & 3.82 & $<0.001$ \\
\hline
\end{tabular}

SF-36v2 The Short Form 36 Health Survey Questionnaire Version 2.0

${ }^{\text {a }}$ Good sleepers were defined as with CSHQ total score lower than 48. Bad sleepers were defined as with CSHQ total score higher than 48.

Table 5 Linear regression analysis of CSHQ score associated with parental PCS and MCS score 
Linear regression Model 1

nnal PCS as dependent value

$\begin{array}{llllllllllll}\text { Constant } & 70.716 & 6.930 & & 10.204 & <0.001 & 0.053 & 65.456 & 4.214 & 15.533 & <0.001 & 0.041 \\ \text { Child's age } & -0.931 & 0.544 & -0.091 & -1.713 & 0.088 & 0.414 & 0.416 & 0.054 & 0.996 & 0.320 \\ & & & & & & & -0.025 & 0.077 & -0.158 & -2.935 & 0.004 \\ \text { Paternal age } & -0.055 & 0.142 & -0.021 & -0.389 & 0.697 & -0.225 & -0.150 & 0.042 & -0.193 & -3.570 & <0.001 \\ \text { Paternal ZBI score } & 0.054 & 0.036 & 0.079 & 1.509 & 0.132 & -0.037 & 0.057 & -0.034 & -0.637 & 0.525 & \end{array}$

nnal MCS as dependent value

$\begin{array}{llllllllllllll}\text { Constant } & 33.761 & 13.915 & & 2.426 & 0.016 & 0.016 & 61.335 & 5.925 & 10.352 & <0.001 & 0.102 \\ \text { Child's age } & -0.935 & 1.091 & -0.046 & -0.857 & 0.392 & & 0.072 & 0.585 & 0.007 & 0.124 & 0.901 \\ \text { Paternal age } & 0.186 & 0.285 & 0.035 & 0.653 & 0.514 & -0.125 & 0.108 & -0.061 & -1.163 & 0.246 \\ \text { Paternal ZBI score } & 0.201 & 0.072 & 0.148 & 2.789 & 0.006 & -0.364 & 0.059 & -0.325 & -6.165 & <0.001 \\ \text { CSHQ total score } & -0.245 & 0.174 & -0.075 & -1.412 & 0.159 & -0.062 & 0.081 & -0.041 & -0.772 & 0.441 & \end{array}$

:rnal PCS as dependent value

\begin{tabular}{lllllllllllll} 
Constant & 59.891 & 6.467 & & 9.261 & $<0.001$ & 0.043 & 60.269 & 5.077 & 11.871 & $<0.001$ & 0.066 \\
Child's age & -0.345 & 0.496 & -0.034 & -0.694 & 0.488 & -0.146 & 0.479 & -0.016 & -0.306 & 0.760 \\
Maternal age & 0.141 & 0.151 & 0.045 & 0.935 & 0.350 & -0.079 & 0.107 & -0.039 & -0.733 & 0.464 \\
\hline Maternal ZBI score & -0.114 & 0.035 & -0.156 & -3.295 & 0.001 & -0.227 & 0.044 & -0.274 & -5.136 & $<0.001$ \\
CSHQ total score & -0.234 & 0.077 & -0.143 & -3.029 & 0.003 & 0.003 & 0.067 & 0.003 & 0.049 & 0.961 &
\end{tabular}

:rnal MCS as dependent value

\begin{tabular}{|c|c|c|c|c|c|c|c|c|c|c|c|c|}
\hline Constant & 48.472 & 9.063 & & 5.348 & $<0.001$ & 0.084 & 80.552 & 6.781 & & 11.879 & $<0.001$ & 0.226 \\
\hline Child's age & 0.746 & 0.696 & 0.051 & 1.073 & 0.284 & & -0.637 & 0.640 & -0.048 & -0.995 & 0.321 & \\
\hline Maternal age & -0.064 & 0.211 & -0.014 & -0.305 & 0.761 & & -0.284 & 0.143 & -0.095 & -1.984 & 0.048 & \\
\hline Maternal ZBI score & -0.305 & 0.048 & -0.292 & -6.301 & $<0.001$ & & -0.517 & 0.059 & -0.425 & -8.756 & $<0.001$ & \\
\hline CSHQ total score & -0.140 & 0.108 & -0.060 & -1.293 & 0.197 & & -0.252 & 0.089 & -0.138 & -2.831 & 0.005 & \\
\hline
\end{tabular}

Linear regression Model 2

nnal PCS as dependent value

\begin{tabular}{lccccll} 
Constant & 73.130 & 7.153 & & 10.224 & $<0.001$ & 0.043 \\
Child's age & -0.896 & 0.544 & -0.088 & -1.649 & 0.100 & \\
\hline Paternal age & -0.053 & 0.142 & -0.020 & -0.371 & 0.711 \\
\hline Paternal ZBI score & 0.061 & 0.036 & 0.089 & 1.690 & 0.092 \\
SRS total score & -0.038 & 0.028 & -0.071 & -1.341 & 0.181 \\
CSHQ total score & -0.319 & 0.087 & -0.194 & -3.651 & $<0.001$
\end{tabular}

rnal MCS as dependent value

\begin{tabular}{lcccccc} 
Constant & 40.953 & 14.317 & & 2.860 & 0.004 & 0.025 \\
Child's age & -0.833 & 1.088 & -0.041 & -0.765 & 0.445 \\
\hline Paternal age & 0.194 & 0.284 & 0.037 & 0.684 & 0.494 \\
\hline Paternal ZBI score & 0.222 & 0.073 & 0.163 & 3.062 & 0.002 \\
SRS total score & -0.113 & 0.057 & -0.107 & -1.996 & 0.047 \\
\hline CSHQ total score & -0.193 & 0.175 & -0.059 & -1.105 & 0.270
\end{tabular}


:rnal PCS as dependent value

\begin{tabular}{|c|c|c|c|c|c|c|}
\hline Constant & 59.516 & 6.654 & & 8.944 & $<0.001$ & 0.041 \\
\hline Child's age & -0.345 & 0.497 & -0.034 & -0.695 & 0.488 & \\
\hline Maternal age & 0.140 & 0.151 & 0.045 & 0.930 & 0.353 & \\
\hline Maternal ZBI score & -0.116 & 0.036 & -0.159 & -3.248 & 0.001 & \\
\hline SRS total score & .006 & 0.026 & .012 & 0.244 & 0.807 & \\
\hline CSHQ total score & -0.237 & 0.078 & -0.145 & -3.030 & 0.003 & \\
\hline
\end{tabular}

irnal MCS as dependent value

\begin{tabular}{|c|c|c|c|c|c|c|}
\hline \multirow[b]{2}{*}{ Child's age } & 52.956 & 9.280 & & 5.706 & $<0.001$ & 0.091 \\
\hline & 0.754 & 0.693 & 0.051 & 1.087 & 0.277 & \\
\hline Maternal age & -0.056 & 0.210 & -0.012 & -0.266 & 0.790 & \\
\hline Maternal ZBI score & -0.279 & 0.050 & -0.267 & -5.600 & $<0.001$ & \\
\hline SRS total score & -0.075 & 0.036 & -0.100 & -2.089 & 0.037 & \\
\hline CSHQ total score & -0.109 & 0.109 & -0.046 & -0.996 & 0.320 & \\
\hline
\end{tabular}

SRS, Social Responsiveness Scales; CSHQ, Children's Sleep Habits Questionnaire; ZBI, The Zarit Caregiver Burden Interview; SF-36v2 The Short Form 36 Health Survey Questionnaire Version 2.0; PCS, physical health summary; MCS, mental health summary

\section{Figures}

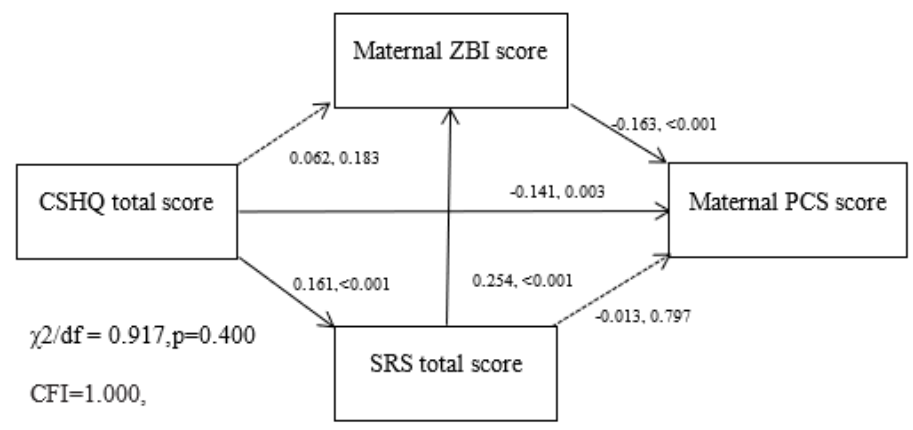

$\mathrm{NFI}=0.973$,

$m+\cdots$

\section{Figure 1}

Pathway analysis of associations among sleep CSHQ score, SRS score, ZBI score and maternal PCS score in ASD group. Significantly pathway in the model showed in bold line.

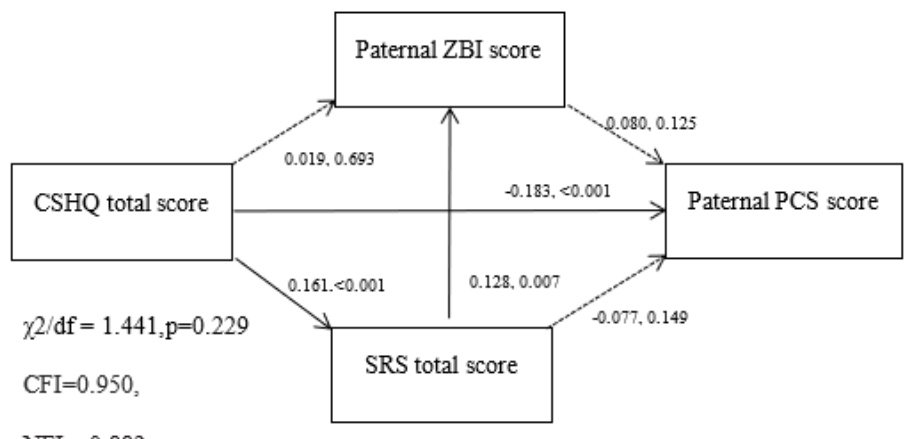

$\mathrm{NFI}=0.882$,

\section{Figure 2}


Pathway analysis of associations among sleep CSHQ score, SRS score, ZBI score and paternal PCS score in ASD group.Significantly pathway in the model showed in bold line.

\section{Supplementary Files}

This is a list of supplementary files associated with this preprint. Click to download.

- TableS1.docx 